\title{
Activation of mineralocorticoid receptors in the rostral ventrolateral medulla is involved in hypertensive mechanisms in stroke-prone spontaneously hypertensive rats
}

\author{
Toshiaki Nakagaki $^{1,2}$, Yoshitaka Hirooka ${ }^{3}$, Ryuichi Matsukawa ${ }^{1}$, Masaaki Nishihara ${ }^{1}$, Masatsugu Nakano ${ }^{1}$, \\ Koji Ito ${ }^{1}$, Sumio Hoka ${ }^{2}$ and Kenji Sunagawa ${ }^{1}$
}

\begin{abstract}
Mineralocorticoid receptor (MR) is recognized as a target for therapeutic intervention in hypertension and heart failure. MRs in the central nervous system are thought to have an important role in blood pressure regulation. Thus, we examined whether activation of the MR pathway in the rostral ventrolateral medulla (RVLM) of the brainstem contributes to the neural mechanism of hypertension in stroke-prone spontaneously hypertensive rats (SHRSPs). We microinjected eplerenone, aldosterone or $\mathrm{Na}^{+}$-rich artificial cerebrospinal fluid (aCSF) into the RVLM of anesthetized Wistar-Kyoto (WKY) rats and SHRSPs. Arterial pressure (AP), heart rate (HR) and renal sympathetic nerve activity (RSNA) were recorded. The expressions of the MR protein and the serumand glucocorticoid-regulated kinase protein (Sgk1), which is a marker of MR activity, in the RVLM were measured by western blot analysis. Bilateral microinjection of eplerenone into the RVLM decreased AP and RSNA in WKY rats and SHRSPs, and the decreases in those variables were significantly greater in SHRSPs than WKY rats. Microinjection of aldosterone or $\mathrm{Na}^{+}$-rich aCSF into the RVLM increased AP and RSNA dose-dependently. The increases in those variables were significantly greater in SHRSPs than in WKY rats. The pressor responses of aldosterone or $\mathrm{Na}^{+}$-rich aCSF were attenuated by the prior injection of eplerenone in SHRSPs. Sgk1 expression levels in the RVLM were significantly greater in SHRSPs than in WKY rats. These findings suggest that activation of MRs in the RVLM enhances sympathetic activity, thereby contributing to the neural mechanism of
\end{abstract} hypertension in the SHRSP.

Hypertension Research (2012) 35, 470-476; doi:10.1038/hr.2011.220; published online 12 January 2012

Keywords: blood pressure; mineralocorticoid receptor; rostral ventrolateral medulla; sympathetic nervous system

\section{INTRODUCTION}

Accumulating evidence indicates that the sympathetic nervous system has an important role in the pathogenesis of hypertension. ${ }^{1-3}$ Mineralocorticoid receptor (MR) is recognized as a target for therapeutic intervention in hypertension and heart failure. ${ }^{4,5}$ The beneficial effects of the MR antagonist spironolactone or the more specific antagonist eplerenone on end-organ damage have been studied in animal models. ${ }^{6-10}$ It has been reported that MRs regulate epithelial $\mathrm{Na}$ channels $(\mathrm{ENaCs})$ that are important for the regulation of sodium transport and the maintenance of extracellular fluid volume and arterial pressure (AP) in the kidney. ${ }^{11}$ Recent studies have reported the distribution of MRs or ENaCs in the choroid plexus, ependyma and neurons, such as those in the supraoptic nucleus, paraventricular nucleus and nucleus tractus solitarius. ${ }^{12,13}$ In addition, the expression of MR mRNA has been reported in the brainstem, ${ }^{14}$ including the rostral ventrolateral medulla (RVLM) and caudal ventrolateral medulla. ${ }^{15,16}$ These findings suggest that MRs in the central nervous system, especially in neurons, regulate $\mathrm{Na}^{+}$influx via ENaCs, leading to hypertension and sympathoexcitation. However, it remains to be determined whether activation of MRs in the brainstem contributes to blood pressure regulation via the sympathetic nervous system.

The RVLM of the brainstem is one of the most important vasomotor centers that determines sympathetic nervous system activity, and it is essential for the maintenance of basal vasomotor tone. ${ }^{3,17,18}$ There is a evidence that increased activity of the RVLM leads to sympathetic hyperactivity in various model of hypertension. ${ }^{18-20}$ For example, both the pressor response to the microinjection of angiotensin II into the RVLM and the stimulation of $\mathrm{AT}_{1}$

${ }^{1}$ Department of Cardiovascular Medicine, Kyushu University Graduate School of Medical Sciences, Fukuoka, Japan; ${ }^{2}$ Department of Anesthesiology and Critical Care Medicine, Kyushu University Graduate School of Medical Sciences, Fukuoka, Japan and ${ }^{3}$ Department of Advanced Cardiovascular Regulation and Therapeutics, Kyushu University Graduate School of Medical Sciences, Fukuoka, Japan

Correspondence: Dr Y Hirooka, Department of Advanced Cardiovascular Regulation and Therapeutics, Kyushu University Graduate School of Medical Sciences, 3-1-1 Maidashi, Higashi-ku, Fukuoka 812-8582, Japan.

E-mail: hyoshi@cardiol.med.kyushu-u.ac.jp

Received 16 September 2011; revised 9 October 2011; accepted 17 October 2011; published online 12 January 2012 
receptors were enhanced in spontaneously hypertensive rats (SHRs). ${ }^{21}$ The activation of endogenous $\mathrm{AT}_{1}$ receptors has also been demonstrated in SHRs and stroke-prone spontaneously hypertensive rats (SHRSPs). ${ }^{22,23}$ It has been reported that microinjection of aldosterone into the RVLM increases AP, and that the MR blocker spironolactone decreases $\mathrm{AP}^{24}$ The purpose of the present study was thus to determine whether the MR pathway in the RVLM contributes to the neural regulation of blood pressure through the sympathetic nervous system, and whether the activation of endogenous MRs in the RVLM leads to sympathetic hyperactivity and hypertension in SHRSPs. Furthermore, we examined neuronal sensitivity to $\mathrm{Na}^{+}$in the RVLM, because activation of MRs would induce ENaC upregulation. ${ }^{11}$ SHRSPs are widely used as a model of hypertension, because they develop exaggerated blood pressure elevation with high sympathetic nervous system activity, thereby causing stoke and death. ${ }^{23}$ In addition, oral administration of eplerenone prevented salt-induced cardiac fibrosis in SHRSPs. ${ }^{6}$ For this purpose, we investigated the effects of aldosterone, $\mathrm{Na}^{+}$-rich artificial cerebrospinal fluid (aCSF) and the MR blocker eplerenone administered into the RVLM on arterial blood pressure, heart rate (HR) and renal sympathetic nerve activity (RSNA). The expression of MRs and serum- and glucocorticoidregulated kinase (Sgk1) expression levels, which indicate MR activity, were also evaluated.

\section{METHODS}

This study was reviewed and approved by the Animal Experiments Ethics Committee, Kyushu University Graduate School of Medical Sciences, and was conducted according to the Guidelines for Animal Experiments of Kyushu University.

\section{Animals and general procedures}

Male Wistar-Kyoto rats (WKY/Izm) and SHRSP/Izm (12-16 weeks old; SLC Japan, Hamamatsu, Japan) were used. Food and tap water were available ad libitum throughout the study. The rats were kept in a temperature- and humidity-controlled room with a 12-h light period between 0800 hours to 2000 hours.

\section{Microinjection into the RVLM and recording of blood pressure, HR and RSNA}

SHRSPs and WKY rats were initially anesthetized with sodium pentobarbital ( $50 \mathrm{mg} \mathrm{kg}^{-1}$ i.p., followed by a maintenance dosage of $20 \mathrm{mg} \mathrm{kg}^{-1}$ per h i.v.). A catheter was inserted into the femoral artery to record AP and HR. A tracheal cannula was connected to a ventilator, and the rats were artificially ventilated. Body temperature was monitored with a rectal thermometer and maintained in the range of $36.5-37.5^{\circ} \mathrm{C}$ with a heating pad. The left renal nerve was exposed using a left retroperitoneal flank incision. A pair of stainless steel bipolar electrodes was placed beneath the renal nerve to record multifiber RSNA. ${ }^{25}$ All signals were recorded on a computer using a PowerLab system (AD Instruments, Nagoya, Japan). The signal from the electrodes was amplified, passed through a band pass filter and then rectified and integrated (resetting every $0.1 \mathrm{~s})$. The rats were placed in a stereotaxic frame, and the dorsal surface of the medulla was surgically exposed to allow for positioning of the microinjection pipettes in the RVLM (with the pipette angled rostrally $18^{\circ} \mathrm{C}, 1.8 \mathrm{~mm}$ lateral, $3.5 \mathrm{~mm}$ below the calamus scriptorius), as previously described. ${ }^{26}$ The microinjections (all microinjections were in a volume of 50-100 nl unless otherwise indicated) into the RVLM were made according to the following protocols: (1) bilateral microinjections of the MR blocker eplerenone (100 pmol each); (2) unilateral microinjection of aldosterone $(10 \mathrm{pmol}-1 \mathrm{nmol})$; (3) unilateral microinjection of aldosterone ( $100 \mathrm{pmol}) 30 \mathrm{~min}$ after bilateral microinjections of eplerenone (100 pmol each); (4) unilateral microinjection of $\mathrm{Na}^{+}$-rich aCSF $(0.15-0.2 \mathrm{M})$; and (5) unilateral microinjection of $\mathrm{Na}^{+}$-rich aCSF $(0.2 \mathrm{M}$ in $50 \mathrm{nl}) 30 \mathrm{~min}$ after bilateral microinjections of eplerenone ( $100 \mathrm{pmol}$ each).

aCSF (which contained (in mmoll ${ }^{-1}$ ) $121 \mathrm{NaCl}, 3.4 \mathrm{KCl}, 1.2 \mathrm{MgCl}_{2}, 0.6$ $\mathrm{NaH}_{2} \mathrm{PO}_{4}, 29 \mathrm{NaHCO}_{3}$ and 3.4 glucose) was used as a vehicle, and $\mathrm{Na}^{+}$-rich
aCSF was prepared by adjusting the $\left[\mathrm{Na}^{+}\right]$of aCSF with additional $\mathrm{NaCl}^{27}$ Aldosterone was obtained from Sigma-Aldrich (St Louis, MO, USA). The MR blocker eplerenone was a gift from the Pfizer Pharmaceutical Company, (New York, NY, USA). Drug doses were based on previous reports ${ }^{24,27}$ or our preliminary experiments. Before microinjection of the drugs, the RVLM was identified by monitoring the mean arterial pressure (MAP) after injecting a small dose $(1 \mathrm{nmol})$ of $\mathrm{L}$-glutamate. For bilateral injections, injections were first made on one side, and then the pipette was moved to the contralateral side; the two injections were made $\sim 3 \mathrm{~min}$ apart.

\section{Western blot analysis for the MR and Sgk1 in the RVLM}

To obtain RVLM tissues, the rats were deeply anesthetized with sodium pentobarbital $\left(100 \mathrm{mg} \mathrm{kg}^{-1}\right.$ i.p. $)$ and transcardially perfused with phosphatebuffered saline. The brain was quickly removed. The RVLM tissue was homogenized and then sonicated in a lysing buffer containing $40 \mathrm{mmoll}^{-1}$ of 4-(2-hydroxyethyl)-1-piperazineethanesulfonic acid (HEPES), 1\% Triton X$100,10 \%$ glycerol, $1 \mathrm{mmoll}^{-1}$ phenylmethanesulfonyl fluoride and 1 protease inhibitor cocktail tablet (Roche Diagnostics, Indianapolis, IN, USA). The tissue lysate was centrifuged at 6000 r.p.m. for $5 \mathrm{~min}$ at $4{ }^{\circ} \mathrm{C}$ in a microcentrifuge. The lysate was collected, and the protein concentration was determined using a bicinchoninic acid protein assay kit (Pierce, Rockford, IL, USA). Aliquots of protein $(50 \mu \mathrm{g})$ from each sample were separated on a $7.5 \%$ sodium dodecyl sulfate-polyacrylamide gel. Subsequently, the separated proteins were transferred onto polyvinylidene difluoride membranes (Immobilon-P membrane; Millipore, Billerica, MA, USA). The membranes were incubated with goat IgG polyclonal antibody against MR (1:1000; Santa Cruz Biotechnology, Santa Cruz, CA, USA), with rabbit IgG polyclonal antibody against SGK1 (Abcam, Tokyo, Japan) and with rabbit IgG polyclonal antibody against glyceraldehyde3-phosphate dehydrogenase (1:1000; Santa Cruz Biotechnology) for 24 to $48 \mathrm{~h}$. The membranes were then washed and incubated with horseradish peroxidaseconjugated horse anti-goat IgG or anti-rabbit antibody (1:10000; Santa Cruz Biotechnology) for $40 \mathrm{~min}$. Immunoreactivity was detected by autoradiography using enhanced chemiluminescence and a western blotting detection kit (Amersham, Piscataway, NJ, USA).

\section{Statistical analysis}

All values are expressed as the mean \pm s.e.m. The changes in MAP, HR and RSNA values during the eplerenone microinjection studies and the MR and Sgk1 receptor expression were compared between SHRSPs and WKY rats using an unpaired $t$-test. Intergroup differences in MAP and RSNA after aldosterone and $\mathrm{Na}^{+}$-rich aCSF microinjection were compared using two-way analysis of variance. $P$ values of $<0.05$ were considered statistically significant.

\section{RESULTS}

Effect of blockade of MR in the RVLM on arterial pressure, HR and RSNA

The basal MAP and HR were significantly higher in SHRSPs than in WKY rats $(183.1 \pm 4.1$ vs. $103.2 \pm 2.7 \mathrm{~mm} \mathrm{Hg}, 355.7 \pm 3.1$ vs. $318.1 \pm 3.3$ b.p.m., $P<0.01, n=5$ for each). Bilateral microinjection of the MR blocker eplerenone into the RVLM induced a significant decrease in MAP, HR and RSNA in both SHRSPs and WKY rats. The magnitudes of the decreases in AP and RSNA were significantly greater in SHRSPs than in WKY rats $(\triangle \mathrm{MAP},-31.5 \pm 2.7 v s$. $-12.0 \pm 1.2 \mathrm{~mm} \mathrm{Hg}, P<0.01$; RSNA $\Delta$ baseline, $-14.6 \pm 2.1$ vs. -4.3 $\pm 0.5 \%, P<0.01, n=5$ for each; Figures $1 \mathrm{a}$ and $\mathrm{b})$. In contrast, the decrease in HR was not significantly different between SHRSPs and WKY rats $(\Delta \mathrm{HR},-22.9 \pm 7.8 v$ s. $-17.4 \pm 9.2$ b.p.m.; NS, $n=5)$. These changes occurred several minutes after injection, peaked at 40-60 min, and gradually recovered over time, but they lasted more than $2 \mathrm{~h}$.

\section{Effect of microinjection of aldosterone into the RVLM on arterial} pressure, HR and RSNA

The basal MAP and HR were significantly higher in SHRSPs than in WKY rats $(185.7 . \pm 5.6$ vs. $91.9 \pm 2.7 \mathrm{~mm} \mathrm{Hg}, 356.5 \pm 4.2$ vs. 


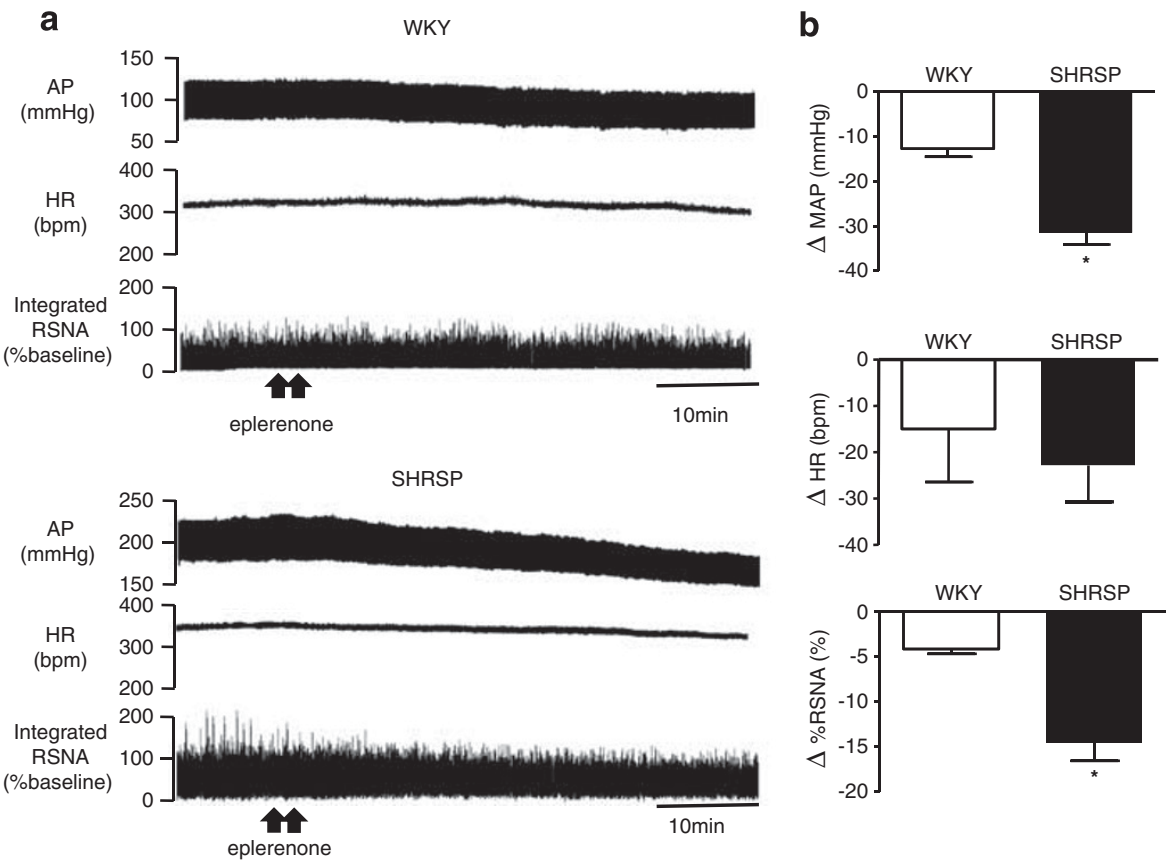

Figure 1 The responses of arterial pressure (AP), heart rate (HR) and renal sympathetic nerve activity (RSNA) to bilateral microinjection of the mineralocorticoid receptor (MR) blocker eplerenone into the RVLM of Wistar-Kyoto (WKY) rats and stroke-prone spontaneously hypertensive rats (SHRSPs). (a) Raw data of the changes in AP, HR and RSNA evoked by bilateral microinjection of eplerenone in WKY rats and SHRSPs. (b) Group data of the changes in mean arterial pressure (MAP), HR and RSNA in response to bilateral microinjection of eplerenone (100 pmol). Values are expressed as the mean \pm s.e.m. $* P<0.05$ ( $n=5$ for each).

a

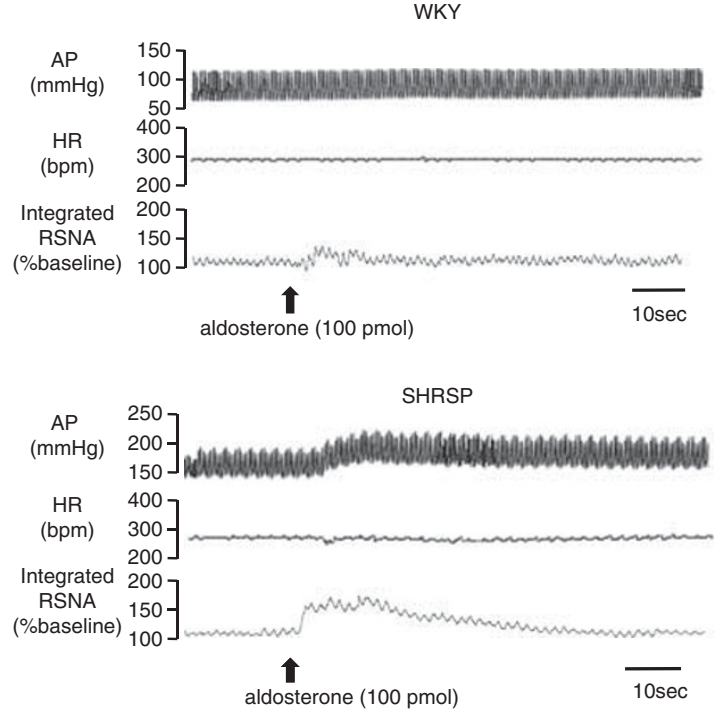

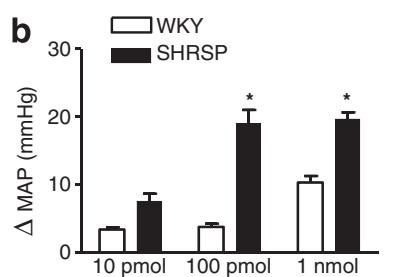
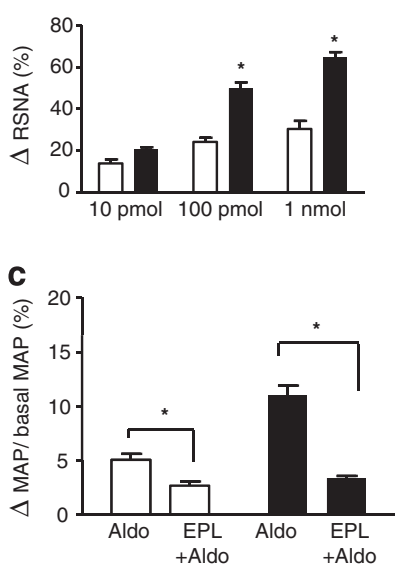

Figure 2 The responses of arterial pressure (AP), heart rate (HR) and renal sympathetic nerve activity (RSNA) to microinjection of aldosterone into the rostral ventrolateral medulla (RVLM). (a) Raw data of the changes in AP, HR and RSNA after unilateral injection of aldosterone (10 pmol-1 nmol) into the RVLM in Wistar-Kyoto (WKY) rats and stroke-prone spontaneously hypertensive rats (SHRSPs). (b) Group data of the changes in mean arterial pressure (MAP) and HR evoked by unilateral microinjection of aldosterone (10 pmol-1 nmol) into the RVLM in WKY rats and SHRSPs. (c) Group data of the changes in MAP in response to microinjection of aldosterone $(100 \mathrm{pmol})$ without pretreatment (Aldo) and microinjection of aldosterone with pretreatment of eplerenone $(100 \mathrm{pmol})\left(\right.$ Aldo + EPL) in WKY rats and SHRSPs. Values are expressed as the mean \pm s.e.m. ${ }^{*} P<0.05$ ( $n=4$ for each). 
$302 \pm 5.1$ b.p.m., $P<0.01, n=4$ for each). Unilateral microinjection of aldosterone into the RVLM significantly increased MAP and RSNA in both SHRSPs and WKY rats. The pressor response induced by aldosterone occurred in a dose-dependent manner (Figures 2a and b). No significant changes in HR were observed in either strain of rats (data not shown). The magnitude of the increases in these variables was significantly greater in SHRSPs than in WKY rats $(P<0.05, n=4$ for each; Figure $2 b)$. Pretreatment with eplerenone nearly prevented the aldosterone-induced pressor responses in SHRSPs and WKY rats. However, the blocking effect of MRs was significantly greater in SHRSPs $(P<0.05, n=5$ for each; Figure $2 \mathrm{c})$.
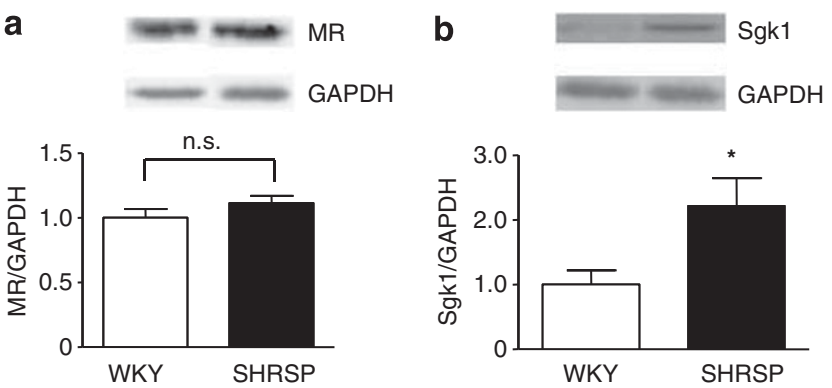

Figure 3 Western blot analysis demonstrating (a) mineralocorticoid receptor (MR) and (b) serum- and glucocorticoid-regulated kinase (Sgk1) expression in the rostral ventrolateral medulla (RVLM) in 12-week-old Wistar-Kyoto (WKY) rats and stroke-prone spontaneously hypertensive rats (SHRSPs). The densitometric average was normalized to the values obtained from the analysis of glyceraldehyde-3-phosphate dehydrogenase (GAPDH) as an internal control. Expressions are shown relative to those seen in WKY rats, which were assigned a value of 1 . Values are expressed as the mean \pm s.e.m. ${ }^{*} P<0.05$ ( $n=4$ for each).

\section{MR and Sgk1 expression levels in the RVLM}

The protein expression levels of MRs in the RVLM did not differ between SHRSPs and WKY rats. However, the levels of Sgk1, which is induced by MRs and is a marker of the activity of MRs, were significantly greater in SHRSPs than in WKY rats $(P<0.05, n=4$ for each; Figure 3).

\section{Effect of microinjection of $\mathrm{Na}^{+}$-rich aCSF into the RVLM on arterial pressure, HR and RSNA}

Similarly, the basal MAP and HR were significantly higher in SHRSPs than in WKY rats $(180.8 . \pm 4.1$ vs. $87.1 \pm 9.9 \mathrm{~mm} \mathrm{Hg}, 357.9 \pm 5.6$ vs. $312.5 \pm 6.4$ b.p.m., $P<0.01, n=5$ for each). Microinjection of $\mathrm{Na}^{+}$-rich aCSF into the RVLM increased both MAP and RSNA in a dosedependent manner (Figures $4 \mathrm{a}$ and $\mathrm{b}$ ), whereas microinjection of $0.15 \mathrm{M}$ aCSF into the RVLM caused no significant changes in MAP, HR or RSNA. The magnitudes of the increases in these variables were significantly greater in SHRSPs than in WKY rats $(P<0.05, n=5$ for each; Figure $4 \mathrm{~b}$ ). In addition, pretreatment with bilateral microinjection of eplerenone into the RVLM significantly attenuated the $\mathrm{Na}^{+}$rich aCSF-induced pressor responses in SHRSPs, but did not significantly change them in WKY rats $(P<0.05, n=5$ for each; Figure 4c).

\section{DISCUSSION}

The findings of this study were as follows: (1) blockade of MRs in the RVLM decreased MAP and RSNA in both SHRSPs and WKY rats, but the decreases were apparently greater in SHRSPs than in WKY rats; (2) microinjection of aldosterone or $\mathrm{Na}^{+}$-rich aCSF into the RVLM increased MAP via sympathetic nerve activity in both SHRSPs and WKY rats, but the increases were greater in SHRSPs than in WKY rats; a

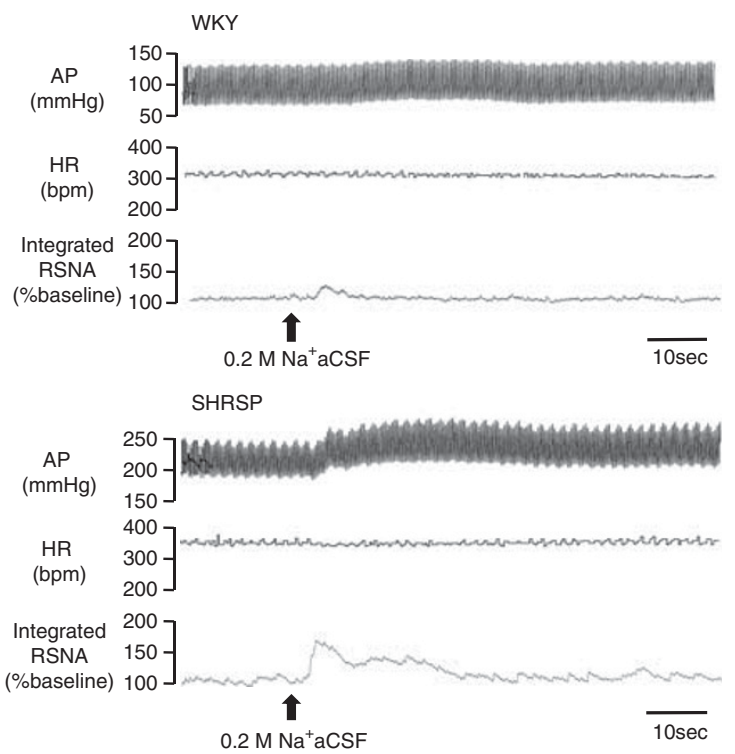

b
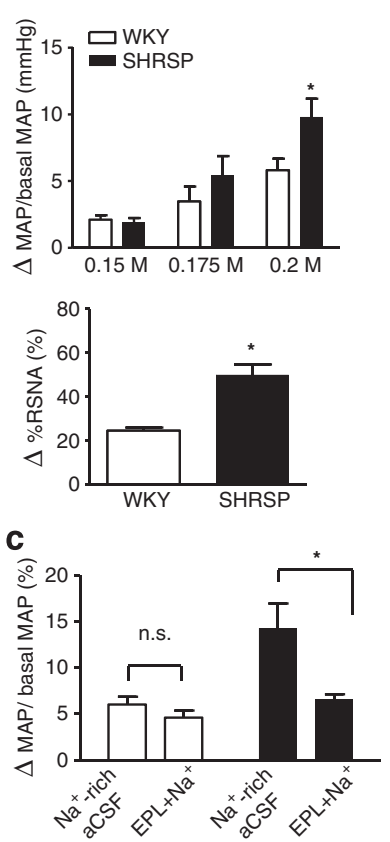

Figure 4 The response to unilateral microinjection of $\mathrm{Na}^{+}$-rich artificial cerebrospinal fluid (aCSF) $(0.15-0.2 \mathrm{M})$ into the rostral ventrolateral medulla (RVLM). (a) Raw data of the changes in mean arterial pressure (AP), heart rate (HR) and renal sympathetic nerve activity (RSNA) in Wistar-Kyoto (WKY) rats and stroke-prone spontaneously hypertensive rats (SHRSPs). (b) Group data of the changes in mean arterial pressure (MAP) and renal sympathetic nerve activity (RSNA) evoked by unilateral microinjection of $\mathrm{Na}^{+}$-aCSF $(0.2 \mathrm{M})$ into the RVLM in WKY rats and SHRSPs. (c) Group data of the changes in mean MAP in response to microinjection of $\mathrm{Na}^{+}$-rich-aCSF $(0.2 \mathrm{M})$ without pretreatment $\left(\mathrm{Na}^{+}\right.$-rich aCSF) and microinjection of $\mathrm{Na}^{+}$rich-aCSF $(0.2 \mathrm{M})$ with pretreatment of eplerenone $(100 \mathrm{pmol})(\mathrm{EPL}+\mathrm{Na})$ in WKY rats and SHRSPs. Values are expressed as the mean \pm s.e.m. ${ }^{*} P<0.05(n=5$ for each). 
(3) in SHRSPs, the prior injection of an MR blocker completely prevented the aldosterone-induced pressor response, but it only partially prevented the $\mathrm{Na}^{+}$-rich aCSF-induced pressor response; and (4) the protein expression levels of MRs in the RVLM did not differ between SHRSPs and WKY rats. However, the Sgk1 expression levels were significantly greater in SHRSPs than in WKY rats. Taken together, our findings indicate that activation of MRs in the RVLM contributes to the neural mechanisms of hypertension in SHRSPs.

The most important finding of this study was that microinjection of the MR-specific blocker eplerenone into the RVLM induced decreases in MAP and RSNA in WKY rats and SHRSPs, and these decreases were greater in SHRSPs. MRs in the brain have been shown to be involved in sympathetic nerve activation in salt-sensitive hypertensive rats, ${ }^{28}$ in a rat model of myocardial infarction ${ }^{29}$ and in other saltsensitive models. ${ }^{30}$ The blockade of MRs in the brain has been suggested to decrease sympathetic nerve activation and result in a decrease in the blood pressure rise from high salt intake in saltsensitive rats. ${ }^{28}$ This decrease has also been associated with an improvement in the cardiac function of mice with pressure overload. ${ }^{30}$ In the present study, we confirmed that endogenous blockade of MRs in the RVLM decreased blood pressure via sympathoinhibition, with greater decreases in SHRSPs. However, the decreases in HR were not significantly different, because it is difficult to assess HR in an acute experiment with anesthetics. To investigate the long-term effects of endogenous MR blockade on blood pressure or RSNA, we microinjected eplerenone into the RVLM bilaterally. It has been reported that Sgk1 is induced by aldosterone and upregulates ENaC levels and activity in the kidney, ${ }^{11,31}$ and that this aldosterone-induced upregulation is dependent on MRs. ${ }^{32}$ Therefore, these studies suggest that Sgk1 can be classified as an aldosterone-effector kinase and a marker of MR signaling. In the RVLM, the protein expression of MRs did not differ between the two strains, but Sgk1 was significantly higher in SHRSPs than in WKY rats. The enhanced protein expression of Sgk1 in SHRSPs in the present study might indicate an enhancement of MR stimulation in the RVLM. We observed that endogenous blockade of MRs by eplerenone induced a greater depressor response through sympathoinhibition in SHRSPs than in WKY rats, and exogenous stimulation of MR by aldosterone elicited a greater pressor response through sympathoexcitation in SHRSPs than in WKY rats. Altogether, these findings suggest that activation of MRs in the RVLM is enhanced and contributes to elevation of the AP in SHRSPs.

The effects of aldosterone have been ascribed to a genomic mechanism of binding to its receptors, followed by translocation of the steroid receptor complex to the nucleus, where it acts as a transcriptional regulator. However, recent studies suggest that effects may be because of non-genomic actions of aldosterone, which occur more rapidly after binding MRs. ${ }^{33,34}$ For example, the rapid action of aldosterone in the kidney ${ }^{33}$ or vasculature ${ }^{34}$ has been demonstrated within a few minutes. Therefore, it is possible that acute inhibition of MR activation might be caused by non-genomic mechanism in the RVLM. However, we cannot exclude the possibility that genomic action is also involved in our observation, because the depressor response evoked by eplerenone lasted more than $2 \mathrm{~h}$. In addition, it should be noted that the depressor and sympathoinhibitory responses occurred several minutes after the bilateral microinjection of eplerenone into the RVLM, and that the pressor and sympathoexcitatory responses occurred within seconds after the microinjection of aldosterone. In general, exogenously administered neurotransmitter/neuromodulators into the RVLM have been shown to elicit rapid action of blood pressure and sympathetic nerve activity. In contrast, the blockade of endogenous receptors in the RVLM gradually evokes the responses of blood pressure and sympathetic nerve activity. ${ }^{35}$ In this context, the time course of the responses is not surprising, although we still do not have a clear explanation for this phenomenon. Further study is needed to clarify whether endogenous activation of MR is involved in hypertensive mechanisms both in the genomic and nongenomic action of aldosterone.

Our observations are consistent with previous reports that microinjection of aldosterone into the RVLM increases MAP. ${ }^{24}$ It has been reported that intracerebroventricular (ICV) infusion of aldosterone increased $\mathrm{MAP}^{36}$ and that these sympathoexcitatory and central pressor effects of aldosterone can be blocked by ICV infusion of an MR blocker. In the present study, microinjection of the MR blocker eplerenone into the RVLM also prevented an aldosterone-induced pressor response. These results suggest that aldosterone is likely to be an endogenous ligand of MRs in the RVLM. It has also been reported that central administration of aldosterone appears to depend on the $\mathrm{MR}-\mathrm{ENaC}$-ouabain pathway and, ultimately, $\mathrm{AT}_{1}$ receptor stimulation. $^{37}$ The sympathoexcitatory and pressor responses to central infusion of aldosterone and $\mathrm{Na}^{+}$-rich aCSF can be prevented by central infusion of the sodium channel blocker benzamil or an ouabain blocker, ${ }^{27,38}$ and the pressor responses elicited by central infusion of aldosterone or ouabain can be blocked by an $\mathrm{AT}_{1}$ blocker. ${ }^{27,39}$ In the RVLM, microinjection of an ouabain-like compound ${ }^{40}$ or angiotensin $\mathrm{II}^{21}$ elicits a pressor response and sympathoexcitation. Collectively, these data suggest that aldosterone in the RVLM might activate central mechanism(s) involving the MR-ENaC-ouabain pathway, thereby causing sympathetic hyperactivity and hypertension.

Several studies have reported that aldosterone shows poor penetration of the blood-brain barrier compared with other steroid hormones. ${ }^{12}$ However, the enzymes for steroid biosynthesis are present in the central nervous system ${ }^{41}$ and RVLM, ${ }^{15}$ and aldosterone can be detected in the tissues of various brain regions in vitro ${ }^{41}$ and in vivo. ${ }^{42}$ In particular, it should be noted that Gomez-Sanchez et al. reported that aldosterone was detectable in the whole brain of adrenalectomized rats, despite the fact that plasma aldosterone was undetectable. ${ }^{41}$ These finding suggest that aldosterone is produced locally in the RVLM. In the present study, such locally produced aldosterone might have been enhanced and might have activated MRs in SHRSPs. Alternatively, there might be an aldosterone-independent MR activation pathway in the RVLM. It has been reported that MRs were activated by Rac1, which was independent of aldosterone in the kidney. ${ }^{43}$ Further studies are needed to clarify the precise mechanisms involved in the activation of MR in the RVLM.

In this study, microinjection of $\mathrm{Na}^{+}$-rich aCSF into the RVLM caused concentration-related increases in MAP and RSNA, whereas microinjection of $0.15 \mathrm{M}$ aCSF at the same volume generated no significant increases. It has been reported that acute ICV infusion ${ }^{44}$ or microinjection into the paraventricular nucleus of $\mathrm{Na}^{+}$-rich aCSF${ }^{27}$ causes sympathetic hyperactivity and hypertension in normotensive rats, and pressor responses are enhanced in salt-sensitive rats compared with salt-resistant rats. ${ }^{44}$ Abrams et al. ${ }^{45}$ hypothesized that when MRs bind ligand, there is a subsequent upregulation of ENaCs, which would increase the membrane permeability to sodium in the brain. This response, in the face of transient increases in sodium levels, would lead to membrane depolarization and an increase in neural activity, driving sympathetic outflow and increasing MAP. It has also been reported that in the paraventricular nucleus, it is possible for an increase in intracellular $\mathrm{Na}^{+}$caused by a larger extra-/intracellular gradient to increase intracellular $\mathrm{Ca}^{2+}$ through the $\mathrm{Na}^{+} / \mathrm{Ca}^{2+}$ channel exchanger, and thereby increase Ang II release. ${ }^{27}$ Considering these 
observations, it is possible that $\mathrm{Na}^{+}$-rich aCSF in the RVLM increases sympathetic nerve activity and MAP.

The pressor effect was greater in SHRSPs than in WKY rats, and this difference between the strains might have been related to the different neural responsiveness to $\mathrm{Na}^{+}$-rich aCSF via ENaCs in the RVLM. This pressor effect was partially blocked by the MR blocker eplerenone in SHRSPs, indicating that the effect of $\mathrm{Na}^{+}$might be mediated by MR activation. ICV infusion of the MR blockers, spironolactone ${ }^{46}$ or benzamil, ${ }^{47}$ prevented $\mathrm{Na}^{+}$-induced sympathoexcitatory and pressor responses in WKY rats. It has also been reported that ICV infusion of eplerenone attenuated $\mathrm{ENaC}$ expression in mice with pressure overload. ${ }^{30}$ Taken together, these findings suggest that MRs mediate $\mathrm{Na}^{+}$ via $\mathrm{ENaCs}$ or transporters on the cell surface of neurons in the RVLM.

\section{Study limitations}

MRs are largely occupied by the glucocorticoid corticosterone, ${ }^{48}$ which is present in a higher concentration than aldosterone in the brain. ${ }^{41}$ The enzyme $11 \beta$-hydroxysteroid dehydrogenase type 2 (11 $\beta$ HSD2), which is distributed the brainstem, including the nucleus tractus solitarius, ${ }^{49}$ rapidly converts corticosterone to an inactive metabolite. Thus, the coexpression of $11 \beta-H S D 2$ with MRs may identify brain regions that are particularly sensitive to aldosterone. Although the precise expression of $11 \beta-H S D 2$ in the RVLM has not yet been determined, we found that aldosterone in the RVLM increased blood pressure, and this pressor response was prevented by the MR blocker eplerenone. Therefore, our findings suggest that aldosterone acts on the MRs in the RVLM. We still cannot exclude the possibility that corticosterone, instead of aldosterone, may act on the MRs in the RVLM. Together with the origin of aldosterone in the RVLM as well as the central nervous system, the study regarding ligand-specifying mechanisms has just begun. In addition, we did not determine whether $\mathrm{ENaC}$ activity is involved in the neural responsiveness to $\mathrm{Na}^{+}$-rich aCSF in the RVLM, because we did not measure $\mathrm{ENaC}$ activation in the RVLM. However, it is possible that MRs and $\mathrm{ENaCs}$ in the RVLM may be involved in this mechanism.

In conclusion, these findings indicate that MRs in the RVLM contribute to the neural mechanisms of hypertension via sympathetic nerve activity, and that increased activity of MRs may be involved in the elevation of blood pressure in SHRSPs.

\section{CONFLICT OF INTEREST}

The authors declare no conflict of interest.

\section{ACKNOWLEDGEMENTS}

We express our sincere thanks to Naomi Shirouzu for help with the Western blot analysis. This study was supported by the Grants-in-Aid for Scientific Research from the Japan Society for the Promotion of Science (B19390231, S23220013) and, in part, by the Salt Science Research Foundation (1034).

1 Esler M. The 2009 Carl Ludwig Lecture: Pathophysiology of the human sympathetic nervous system in cardiovascular diseases: the transition from mechanisms to medical management. J Appl Physiol 2010; 108: 227-237.

2 Grassi G. Assessment of sympathetic cardiovascular drive in human hypertension: achievements and perspectives. Hypertension 2009; 54: 690-697.

3 Guyenet PG. The sympathetic control of blood pressure. Nat Rev Neurosci 2006; 7 335-346.

4 Pitt B, Remme W, Zannad F, Neaton J, Martinez F, Roniker B, Bittman R, Hurley S, Kleiman J, Gatlin M. Eplerenone, a selective aldosterone blocker, in patients with left ventricular dysfunction after myocardial infarction. N Engl J Med 2003; 348 1309-1321.
5 Yagi S, Akaike M, Aihara K, Iwase T, Yoshida S, Sumitomo-Ueda Y, Ikeda Y, Ishikawa K, Matsumoto T, Sata M. High plasma aldosterone concentration is a novel risk factor of cognitive impairment in patients with hypertension. Hypertens Res 2011; 34: 74-78.

6 Endemann DH, Touyz RM, Iglarz M, Savoia C, Schiffrin EL. Eplerenone prevents saltinduced vascular remodeling and cardiac fibrosis in stroke-prone spontaneously hypertensive rats. Hypertension 2004; 43: 1252-1257.

7 Kimura S, Ito M, Tomita M, Hoyano M, Obata H, Ding L, Chinushi M, Hanawa H, Kodama M, Aizawa Y. Role of mineralocorticoid receptor on atrial structural remodeling and inducibility of atrial fibrillation in hypertensive rats. Hypertens Res 2011; 34: 584-591.

8 Min LJ, Mogi M, Iwanami J, Sakata A, Jing F, Tsukuda K, Ohshima K, Horiuchi M. Angiotensin II and aldosterone-induced neuronal damage in neurons through an astrocyte-dependent mechanism. Hypertens Res 2011; 34: 773-778.

9 Nakamura T, Fukuda M, Kataoka K, Nako H, Tokutomi Y, Dong YF, Yamamoto E, Yasuda $\mathrm{O}$, Ogawa H, Kim-Mitsuyama S. Eplerenone potentiates protective effects of amlodipine against cardiovascular injury in salt-sensitive hypertensive rats. Hypertens Res 2011; 34: 817-824.

10 Takeda Y. Effects of eplerenone, a selective mineralocorticoid receptor antagonist, on clinical and experimental salt-sensitive hypertension. Hypertens Res 2009; 32: 321-324.

11 Schild L. The epithelial sodium channel and the control of sodium balance. Biochim Biophys Acta 2010; 1802: 1159-1165.

12 Geerling JC, Loewy AD. Aldosterone in the brain. Am J Physiol Renal Physiol 2009; 297: F559-F576.

13 Amin MS, Wang HW, Reza E, Whitman SC, Tuana BS, Leenen FH. Distribution of epithelial sodium channels and mineralocorticoid receptors in cardiovascular regulatory centers in rat brain. Am J Physiol Regul Integr Comp Physiol 2005; 289: R1787-R1797.

14 Gomez-Sanchez EP, Gomez-Sanchez CM, Plonczynski M, Gomez-Sanchez CE. Aldosterone synthesis in the brain contributes to Dahl salt-sensitive rat hypertension. Exp Physiol 2010; 95: 120-130.

15 Kumar NN, Goodchild AK, Li Q, Pilowsky PM. An aldosterone-related system in the ventrolateral medulla oblongata of spontaneously hypertensive and Wistar-Kyoto rats. Clin Exp Pharmacol Physiol 2006; 33: 71-75.

16 Comer AM, Gibbons HM, Qi J, Kawai Y, Win J, Lipski J. Detection of mRNA species in bulbospinal neurons isolated from the rostral ventrolateral medulla using single-cell RTPCR. Brain Res Brain Res Protoc 1999; 4: 367-377.

17 Sved AF, Ito S, Sved JC. Brainstem mechanisms of hypertension: role of the rostral ventrolateral medulla. Curr Hypertens Rep 2003; 5: 262-268.

18 Hirooka Y. Oxidative stress in the cardiovascular center has a pivotal role in the sympathetic activation in hypertension. Hypertens Res 2011; 34: 407-412.

19 Kishi T, Hirooka Y, Kimura Y, Ito K, Shimokawa H, Takeshita A. Increased reactive oxygen species in rostral ventrolateral medulla contribute to neural mechanisms of hypertension in stroke-prone spontaneously hypertensive rats. Circulation 2004; 109: 2357-2362.

20 Hirooka Y, Sagara Y, Kishi T, Sunagawa K. Oxidative stress and central cardiovascular regulation. - Pathogenesis of hypertension and therapeutic aspects -. Circ J 2010; 74: $827-835$.

21 Ito S, Komatsu K, Tsukamoto K, Kanmatsuse K, Sved AF. Ventrolateral medulla AT1 receptors support blood pressure in hypertensive rats. Hypertension 2002; 40: 552-559.

22 Kishi T, Hirooka Y, Konno S, Ogawa K, Sunagawa K. Angiotensin II type 1 receptoractivated caspase-3 through ras/mitogen-activated protein kinase/extracellular signal-regulated kinase in the rostral ventrolateral medulla is involved in sympathoexcitation in stroke-prone spontaneously hypertensive rats. Hypertension 2010; 55 . 291-297.

23 Yamori Y, Horie R, Handa H, Sato M, Fukase M. Pathogenetic similarity of strokes in stroke-prone spontaneously hypertensive rats and humans. Stroke 1976; 7: 46-53.

24 Zhu DN, Xue LM, Li P. Cardiovascular effects of microinjection of corticoids and antagonists into the rostral ventrolateral medulla in rats. Blood Press 1995; 4: 55-62.

25 Matsukawa R, Hirooka Y, Nishihara M, Ito K, Sunagawa K. Neuregulin-1/ErbB signaling in rostral ventrolateral medulla is involved in blood pressure regulation as an antihypertensive system. J Hypertens 2011; 29: 1735-1742.

26 Koga Y, Hirooka Y, Araki S, Nozoe M, Kishi T, Sunagawa K. High salt intake enhances blood pressure increase during development of hypertension via oxidative stress in rostral ventrolateral medulla of spontaneously hypertensive rats. Hypertens Res 2008; 31: 2075-2083.

27 Gabor A, Leenen FH. Mechanisms in the PVN mediating local and central sodiuminduced hypertension in Wistar rats. Am J Physiol Regul Integr Comp Physiol 2009; 296: R618-R630.

28 Huang BS, White RA, Jeng AY, Leenen FH. Role of central nervous system aldosterone synthase and mineralocorticoid receptors in salt-induced hypertension in Dahl saltsensitive rats. Am J Physiol Regul Integr Comp Physiol 2009; 296: R994-R1000.

29 Huang BS, Leenen FH. Blockade of brain mineralocorticoid receptors or $\mathrm{Na}+$ channels prevents sympathetic hyperactivity and improves cardiac function in rats post-MI. Am J Physiol Heart Circ Physiol 2005; 288: H2491-H2497.

30 Ito K, Hirooka Y, Sunagawa K. Blockade of mineralocorticoid receptors improves saltinduced left-ventricular systolic dysfunction through attenuation of enhanced sympathetic drive in mice with pressure overload. J Hypertens 2010; 28: 1449-1458.

31 Wulff P, Vallon V, Huang DY, VolkI H, Yu F, Richter K, Jansen M, Schlunz M, Klingel K, Loffing J, Kauselmann G, BosI MR, Lang F, Kuhl D. Impaired renal $\mathrm{Na}(+)$ retention in the sgk1-knockout mouse. J Clin Invest 2002; 110: 1263-1268.

32 Shibata S, Nagase M, Yoshida S, Kawachi H, Fujita T. Podocyte as the target for aldosterone: roles of oxidative stress and Sgk1. Hypertension 2007; 49: 355-364. 
33 Zhou ZH, Bubien JK. Nongenomic regulation of ENaC by aldosterone. Am J Physiol Cell Physiol 2001; 281: C1118-C1130.

34 Gros R, Ding Q, Armstrong S, O'Neil C, Pickering JG, Feldman RD. Rapid effects of aldosterone on clonal human vascular smooth muscle cells. Am J Physiol Cell Physiol 2007; 292: C788-C794.

35 Dampney RAL. Functional organization of central pathways regulating the cardiovascular system. Physiol Rev 1994; 74: 323-364.

36 Zhang ZH, Yu Y, Kang YM, Wei SG, Felder RB. Aldosterone acts centrally to increase brain renin-angiotensin system activity and oxidative stress in normal rats. Am J Physiol Heart Circ Physiol 2008; 294: H1067-H1074.

37 Huang BS, Leenen FH. Mineralocorticoid actions in the brain and hypertension. Curr Hypertens Rep 2011; 13: 214-220.

38 Wang H, Huang BS, Leenen FH. Brain sodium channels and ouabainlike compounds mediate central aldosterone-induced hypertension. Am J Physiol Heart Circ Physiol 2003; 285: H2516-H2523.

39 Huang BS, Leenen FH. Sympathoexcitatory and pressor responses to increased brain sodium and ouabain are mediated via brain II ANG. Am J Physiol 1996; 270: $\mathrm{H} 275-\mathrm{H} 280$.

40 Teruya H, Yamazato M, Muratani H, Sakima A, Takishita S, Terano Y, Fukiyama K. Role of ouabain-like compound in the rostral ventrolateral medulla in rats. J Clin Invest 1997; 99: 2791-2798.

41 Gomez-Sanchez EP, Ahmad N, Romero DG, Gomez-Sanchez CE. Is aldosterone synthesized within the rat brain? Am J Physiol Endocrinol Metab 2005; 288: E342-E346.
42 Yu Y, Wei SG, Zhang ZH, Gomez-Sanchez E, Weiss RM, Felder RB. Does aldosterone upregulate the brain renin-angiotensin system in rats with heart failure? Hypertension 2008; 51: 727-733.

43 Shibata S, Nagase M, Yoshida S, Kawarazaki W, Kurihara H, Tanaka H, Miyoshi J, Takai Y, Fujita T. Modification of mineralocorticoid receptor function by Rac1 GTPase: implication in proteinuric kidney disease. Nat Med 2008; 14: 1370-1376.

44 Huang BS, Wang H, Leenen FH. Enhanced sympathoexcitatory and pressor responses to central Na+ in Dahl salt-sensitive vs. -resistant rats. Am J Physiol Heart Circ Physiol 2001; 281: H1881-H1889.

45 Abrams JM, Osborn JW. A role for benzamil-sensitive proteins of the central nervous system in the pathogenesis of salt-dependent hypertension. Clin Exp Pharmacol Physiol 2008; 35: 687-694.

46 Huang BS, Cheung WJ, Wang H, Tan J, White RA, Leenen FH. Activation of brain reninangiotensin-aldosterone system by central sodium in Wistar rats. Am J Physiol Heart Circ Physiol 2006; 291: H1109-H1117.

47 Nishimura M, Ohtsuka K, Nanbu A, Takahashi H, Yoshimura M. Benzamil blockade of brain $\mathrm{Na}+$ channels averts $\mathrm{Na}(+)$-induced hypertension in rats. Am J Physiol 1998; 274: R635-R644.

48 de Kloet ER, Van Acker SA, Sibug RM, Oitzl MS, Meijer OC, Rahmouni K, de Jong W. Brain mineralocorticoid receptors and centrally regulated functions. Kidney Int 2000; 57: 1329-1336.

49 Geerling JC, Kawata M, Loewy AD. Aldosterone-sensitive neurons in the rat central nervous system. J Comp Neurol 2006; 494: 515-527. 